Article

\title{
Characterization of a Novel BmaTX47 Toxin Modulating Sodium Channels: The Crucial Role of Expression Vectors in Toxin Pharmacological Activity
}

\author{
Tian Li ${ }^{1}$, Lingna Xu ${ }^{1}$, Honglian Liu ${ }^{1}$, Yawen He ${ }^{1}$, Songping Liang ${ }^{2}$, Wenxin Li ${ }^{1}{ }^{*}$ \\ and Yingliang $\mathrm{Wu}^{1}{ }^{1, *}$
}

1 State Key Laboratory of Virology, College of Life Sciences, Wuhan University, Wuhan 430072, China; E-Mails: fiona2210@163.com (T.L.); xulingna58@126.com (L.X.); honglianliu2011@whu.edu.cn (H.L.); hesunrise@sohu.com (Y.H.)

2 Key Laboratory of Protein Chemistry and Developmental Biology of the Ministry of Education, College of Life Sciences, Hunan Normal University, Changsha 410081, Hunan, China; E-Mail: liangsp@hunnu.edu.cn

* Authors to whom correspondence should be addressed; E-Mails: liwxlab@whu.edu.cn (W.L.); ylwu@whu.edu.cn (Y.W.); Tel.: +86-27-6875-2831; Fax: +86-27-6875-2146 (W.L. \& Y.W.).

Received: 4 December 2013; in revised form: 30 December 2013 / Accepted: 20 January 2014 / Published: 26 February 2014

\begin{abstract}
Long-chain scorpion toxins with four disulfide bridges exhibit various pharmacological features towards the different voltage-gated sodium channel subtypes. However, the toxin production still remains a huge challenge. Here, we reported the effects of different expression vectors on the pharmacological properties of a novel toxin BmaTX47 from the scorpion Buthus martensii Karsch. The recombinant BmaTX47 was obtained using the expression vector $\mathrm{pET}-14 \mathrm{~b}$ and $\mathrm{pET}-28 \mathrm{a}$, respectively. Pharmacological experiments showed that the recombinant Bm $\alpha \mathrm{TX} 47$ was a new $\alpha$-scorpion toxin which could inhibit the fast inactivation of $\mathrm{rNa}_{\mathrm{v}} 1.2, \mathrm{mNa}_{\mathrm{v}} 1.4$ and $\mathrm{hNa}_{\mathrm{v}} 1.5$ channels. Importantly, the different expression vectors were found to strongly affect BmaTX47 pharmacological activities while toxins were obtained by the same expression and purification procedures. When $10 \mu \mathrm{M}$ recombinant BmaTX47 from the pET-28a vector was applied, the values of $I_{5 \mathrm{~ms}} / I_{\text {peak }}$ for $\mathrm{rNa}_{\mathrm{v}} 1.2, \mathrm{mNa}_{\mathrm{v}} 1.4$ and $\mathrm{hNa}_{\mathrm{v}} 1.5$ channels were $44.12 \% \pm 3.17 \%, 25.40 \% \pm 4.89 \%$ and $65.34 \% \pm 3.86 \%$, respectively, which were better than those values of $11.33 \% \pm 1.46 \%$, $15.96 \% \pm 1.87 \%$ and $5.24 \% \pm 2.38 \%$ for $\mathrm{rNa}_{\mathrm{v}} 1.2, \mathrm{mNa}_{\mathrm{v}} 1.4$ and $\mathrm{hNa}_{\mathrm{v}} 1.5$ channels delayed by $10 \mu \mathrm{M}$ recombinant BmaTX47 from the pET-14b vector. The dose-response experiments
\end{abstract}


further indicated the $\mathrm{EC}_{50}$ values of recombinant $\mathrm{Bm \alpha TX} 47$ from the $\mathrm{pET}-28 \mathrm{a}$ vector were $7262.9 \pm 755.9 \mathrm{nM}$ for $\mathrm{rNa}_{\mathrm{v}} 1.2$ channel and $1005.8 \pm 118.6 \mathrm{nM}$ for hNav1.5 channel, respectively. Together, these findings highlighted the important role of expression vectors in scorpion toxin pharmacological properties, which would accelerate the understanding of the structure-function relationships of scorpion toxins and promote the potential application of toxins in the near future.

Keywords: Buthus martensii Karsch; BmaTX47; recombinant expression; sodium channels; pET-28a vector; $\mathrm{pET}-14 \mathrm{~b}$ vector

\section{Introduction}

Voltage-gated sodium channels (VGSCs) are essential for initiating and propagating action potentials in most electrically excitable cells of multicellular organisms [1]. VGSCs are transmembrane proteins consisting of a pore-forming $\alpha$ subunit and one or two auxiliary $\beta$ subunits. So far, nine $\alpha$-subunit isoforms $\left(\mathrm{Na}_{\mathrm{v}} 1.1-\mathrm{Na}_{\mathrm{v}} 1.9\right)$ and five $\beta$ subunit isoforms $(\beta 1 \mathrm{~B}, \beta 1-\beta 4)$ have been identified in the vertebrates [1,2]. The $\alpha$ subunits are comprised of four homologous domains (DI-DIV), each containing six transmembrane helices (S1-S6) connected by extracellular and intracellular loops of variable sizes. So far, the conformational features of vertebrate VGSCs still remain unclear, and the structural complexity is often deciphered by the specific scorpion toxins and other animal toxins nowadays [3-6].

Scorpion toxins specific for the VGSCs are polypeptides containing 60-70 amino acids, stabilized by four disulfide bonds. Over the past years, these toxins have been proven invaluable to understand the complicated structural and functional features of the VGSCs [7]. Based on the different functions and mechanisms, the scorpion toxins specific for the VGSCs are mainly divided into two groups: $\alpha$-toxins and $\beta$-toxins. The $\alpha$-toxins delay the fast channel inactivation and have minor effects on the voltage-dependence of activation through binding to the sodium channel site 3 [8]. In contrast, the $\beta$-toxins hyperpolarize the activation curve and reduce the sodium peak current through binding to site 4 [7]. Although the $\alpha$-toxins display the conserved tertiary structures, they show the remarkable diversity of sequence and function [8]. Thus, $\alpha$-toxins are mainly divided into three subgroups: classical $\alpha$-toxins predominantly acting on the mammalian VGSCs; insect $\alpha$-toxins mainly acting on the insect VGSCs; and $\alpha$-like toxins acting on both the mammalian and insect VGSCs. In order to investigate their pharmacological functions, $\alpha$-toxins are mainly obtained nowadays by isolation from the scorpion venom and gene engineering. Due to the tiny amount of scorpion crude venom available, only several native $\alpha$-scorpion toxins have been isolated, including LqhII, LqhIII and LqhaIT, etc. [9-14]. The alternative strategy is to obtain the recombinant toxins through gene engineering, such as LqhaIT, $\mathrm{AaH} 2$, Lqh II, BmKM1 and BmaTX14 [15-20]. Significantly different from the successful production of the recombinant short-chain scorpion toxins specific for the potassium channels [21-23], the $\alpha$-scorpion toxins specific for the VGSCs are often difficult to produce due to some unknown factors. For example, the $\alpha$-like toxin LqhIII can only be acquired in the form of the fusion peptide His-apamin-LqhIII rather than only LqhIII [24]. Nowadays, this complexity seriously hinders the development of structure-function relationships of both $\alpha$-scorpion toxins and VGSCs. 
Here, we reported a novel $\alpha$-scorpion toxin BmaTX47 from the scorpion Buthus martensii Karsch, and investigated the effect of expression vector on toxin production and function. It was found that the recombinant BmaTX47 could be obtained using both expression vectors $\mathrm{pET}-14 \mathrm{~b}$ and $\mathrm{pET}-28 \mathrm{a}$ with the same procedures, however, BmaTX47 from the different expression vectors showed the distinct activities on $\mathrm{rNa}_{\mathrm{v}} 1.2, \mathrm{mNa}_{\mathrm{v}} 1.4$ and $\mathrm{hNa}_{\mathrm{v}} 1.5$ channels. These findings highlighted the important role of expression vectors on scorpion toxin functions, which would improve the understanding of the structure-function relationships of scorpion toxins and accelerate toxin application as molecular tools and prospective drugs.

\section{Results and Discussion}

\subsection{Cloning and Sequence Analysis of BmaTX47}

Through the random screening and bioinformatics analysis of the $B$. martensii venom gland cDNA library described previously [23,25-27], we found a new putative $\alpha$-scorpion toxin BmaTX47 which might act on sodium channels. The precursor nucleotide sequence had $369 \mathrm{bp}$ including a 5' untranslated region (UTR), ORF and 3' UTR of $48 \mathrm{bp}, 258 \mathrm{bp}$ and $63 \mathrm{bp}$, respectively. The ORF region encoded a polypeptide of 85 amino acid residues, containing a 19-residue signal peptide and a 66-residue mature peptide (Figure 1A).

Figure 1. Sequence and structural analysis of the $\alpha$-scorpion toxin BmaTX47. (A) The precursor nucleotide and amino acids sequences of BmaTX47. The signal peptide is underlined; (B) Amino acid sequence alignment of BmaTX47 with the known $\alpha$-scorpion toxins including Makatoxin-1 (UniProtKB/Swiss-Prot: P56569.1), BmKM1 (UniProtKB/Swiss-Prot: P45697.2), BjaIT (UniProtKB/Swiss-Prot: Q56TT9.1), LqhII (UniProtKB/Swiss-Prot: P59355.1) and LqhaIT (UniProtKB/Swiss-Prot: P17728.2); (C) Structural modeling and analysis of BmaTX47. The experimental and putative functional residues in LqhII and BmaTX47 were labeled, respectively. The Lys2, Thr57 and Arg58 were functional residues composing the NC-domain, while the residues Phe15, Arg18, Trp38 and Asn44 constituted the Core-domain of LqhII. Accordingly, the residues Arg2, Ile57, Arg58, Ile59 and Arg64 were putative functional residues in NC-domain, while Leu18, Trp38, Tyr42 and Asn44 were possibly important residues in Core-domain of BmaTX47, respectively.

A

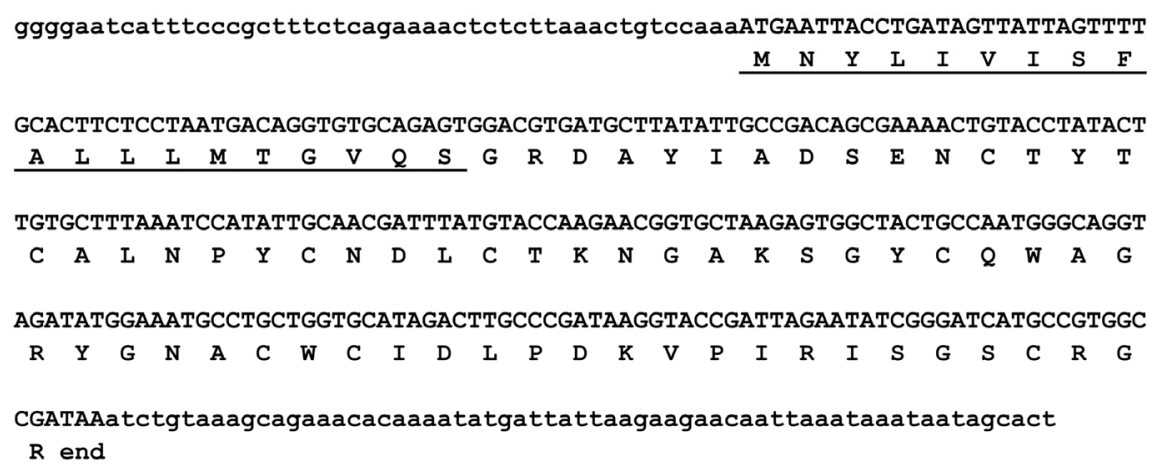


Figure 1. Cont.

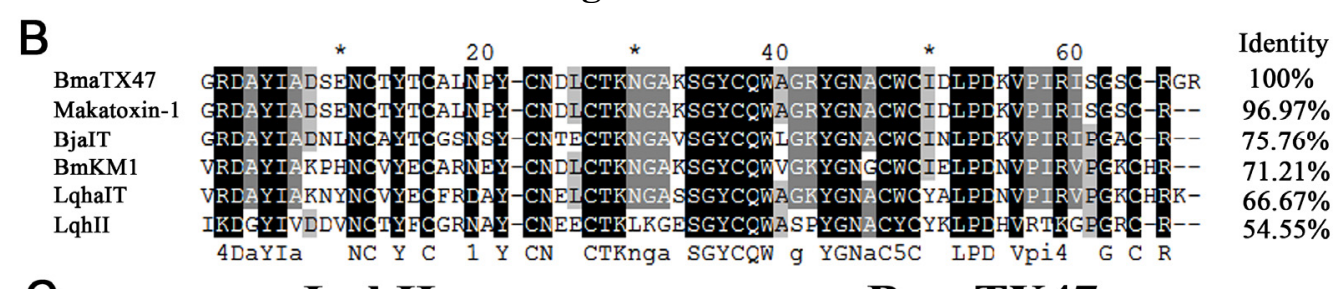

C

LqhII

BmaTX47
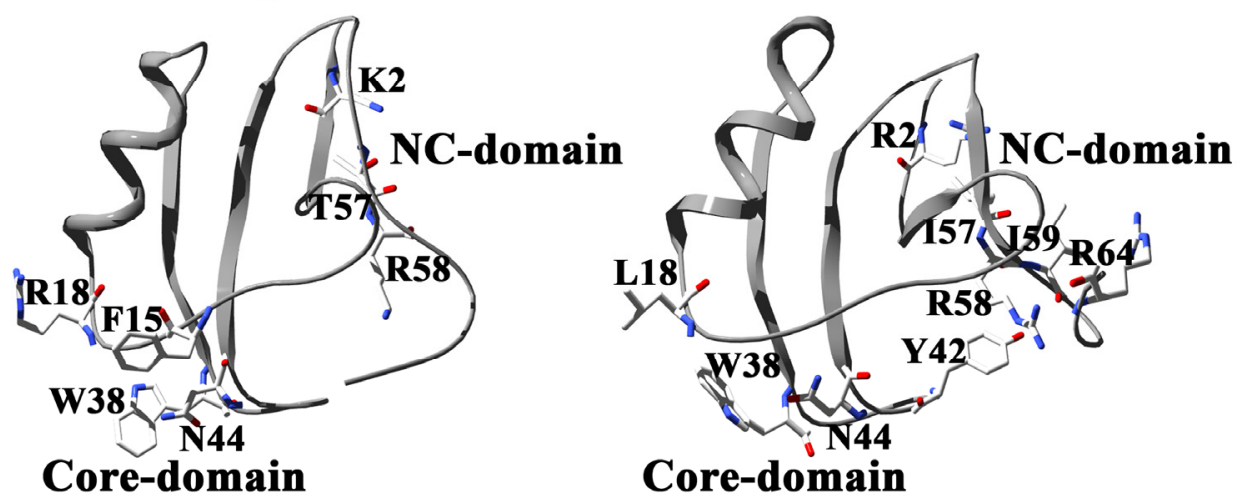

Multiple sequence alignments showed that BmaTX47 shared high homology with other known $\alpha$-scorpion toxins, including Makatoxin-1, BjaIT, BmKM1, LqhaIT, and LqhII (Figure 1B). Since there was no functional information of highly similar toxin Makatoxin-1 on the VGSC subtypes [28], it was essential to characterize the pharmacological function of BmaTX47 in this work. The structural modeling and analysis were performed using $\alpha$-scorpion toxin BmKM1 (PDB code: 1ZYW) as the template. As shown in Figure 1C, BmaTX47 probably adopted the classical structure with an $\alpha$-helix and three antiparallel $\beta$-sheets. Furthermore, the residues Arg2, Ile57, Arg58, Ile59, Arg64 and Arg66 were possible functional residues in the NC-domain, and residues Leu18, Trp38, Tyr42, Asn44 were possible functional residues in the Core-domain of the BmaTX47. Such distribution of potential functional residues resembled that of the classical toxin LqhII $[15,16]$, which suggested that BmaTX47 was an $\alpha$-scorpion toxin specific for the sodium channels.

\subsection{Expression and Purification of Bm $\alpha T X 47$ Using Expression Vector pET-14b}

During the production of the recombinant $\alpha$-scorpion toxins, the expression vector $\mathrm{pET}-14 \mathrm{~b}$ was ever used to express different toxins [15]. In this work, the expression vector $p E T-14 b$ was first used to produce the recombinant BmaTX47. According to our previous method on the recombinant $\alpha$-scorpion toxin BmaTX14 [20], BmaTX47 was inserted into pET-14b between NdeI and BmaHI sites, extending the N-terminus of BmaTX47 with a His ${ }_{6}$-tag and a thrombin cleavage site. The full sequence of the recombinant protein was MGSSHHHHHHSSGLVPRGSHM-GRDAYIADSENCTYTCALNPYCNDL CTKNGAKSGYCQWAGRYGNACWCIDLPDKVPIRISGSCRGR, with the first Met being excluded after translation (Figure 2A). The verified expression vector was subsequently transformed into E. coli Rosetta (DE3) for the toxin expression.

The recombinant BmaTX47 protein was expressed in the inclusion bodies, and was then denatured and refolded in $0.2 \mathrm{M}$ ammonium acetate at $16{ }^{\circ} \mathrm{C}$ according to our previous procedure for the recombinant BmaTX14 toxin [20]. As shown in Figure 2B, the refolded toxin was separated by 
reverse-phase high performance liquid chromatography (RP-HPLC), and the peak at about $20 \mathrm{~min}$ corresponding to rBmaTX47 was manually collected and then analyzed by the sodium dodecyl sulfate polyacrylamide gel electrophoresis (SDS-PAGE) and MALDI-TOF-MS. It could be found that there was only one discernible band at about $9.5 \mathrm{kDa}$ on the analytical gel (Figure 2B), and the determined molecular weight was $9417.7 \mathrm{Da}$, in accordance with the calculated molecular weight 9416.3 Da (Figure 2C). Subsequently, rBmaTX47 was quantified using the BCA Protein Assay kit (Thermo Fisher Scientific, Waltham, MA, USA) and stored at $-80{ }^{\circ} \mathrm{C}$ after the freeze-drying. In previous cases of the recombinant $\alpha$-scorpion toxins, the His $_{6}$-tag was proven to have little effect on the toxin activities $[15,20,24]$, so the tag was not removed in the fusion peptide His ${ }_{6}$-BmaTX47 in this work.

Figure 2. Expression, purification and identification of rBmaTX47 toxin with pET-14b vector. (A) Construction of expression vector BmaTX47-pET-14b. The nucleotide sequence encoding mature BmaTX47 peptide was inserted between NdeI and BamHI sites of the vector $\mathrm{pET}-14 \mathrm{~b}$ downstream of the $\mathrm{His}_{6}$-tag and thrombin cleavage sequence. The full sequence of toxin rBmaTX47 was MGSSHHHHHHSSGLVPRGSHM-BmaTX47; (B) Purification of the refolded $\mathrm{rBm} \alpha \mathrm{TX} 47$ from $\mathrm{pET}-14 \mathrm{~b}$ vector by RP-HPLC together with the tricine-SDS-PAGE analysis; (C) Mass spectrum of rBmaTX47 from the MALDI-TOF-MS. The calculated molecular weight was $9416.3 \mathrm{Da}$, and the measured value was 9417.7 Da.

A

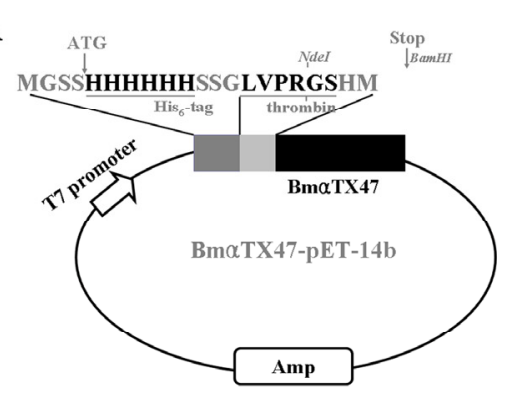

B

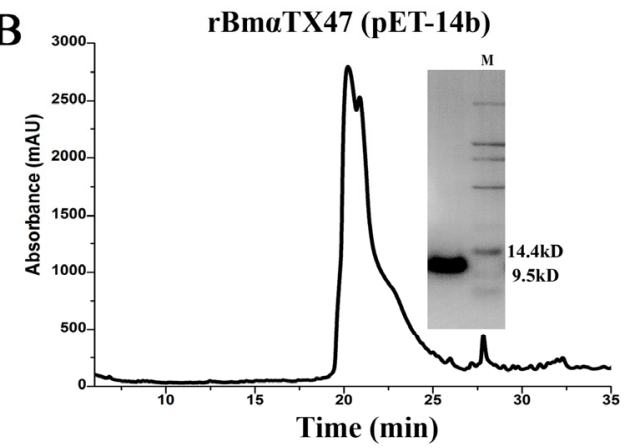

C

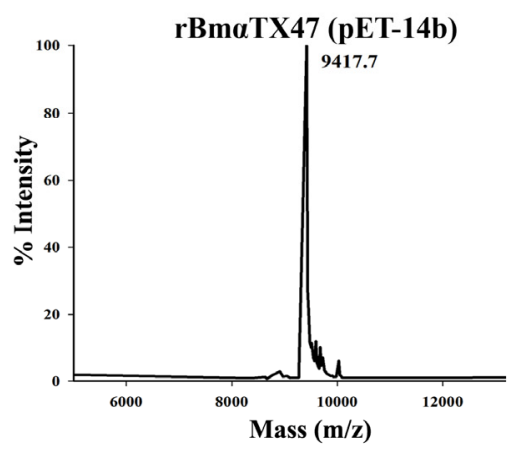

\subsection{Pharmacological Characterization of rBmaTX47 from Expression Vector pET-14b}

The $\mathrm{Na}_{\mathrm{v}} 1.2, \mathrm{Na}_{\mathrm{v}} 1.4$ and $\mathrm{Na}_{\mathrm{v}} 1.5$ sodium channels are widely used to characterize the pharmacological profiles of $\alpha$-scorpion toxins [9,10,15,17,29-34]. In this work, the pharmacological effects of rBmaTX47 from the expression vector pET-14b were also examined on the $\mathrm{rNa}_{\mathrm{v}} 1.2, \mathrm{mNa}_{\mathrm{v}} 1.4$ and $\mathrm{hNa}_{\mathrm{v}} 1.5$ channels expressed in HEK293 cells. As shown in Figure 3 and Table 1, the $10 \mu \mathrm{M} \mathrm{rBm} \alpha \mathrm{TX} 47$ could not efficiently inhibit the fast inactivation of all these channels and the $I_{5 \mathrm{~ms}} / I_{\text {peak }}$ values were $11.33 \% \pm 1.46 \%$ and $15.96 \% \pm 1.87 \%$ for $\mathrm{rNa}_{\mathrm{v}} 1.2$ and $\mathrm{mNa}_{\mathrm{v}} 1.4$ channels, respectively. Meanwhile, the $10 \mu \mathrm{M} \mathrm{rBm} \alpha \mathrm{TX} 47$ was also found to have little effect on the currents of $\mathrm{hNa}_{\mathrm{v}} 1.5$ channel (Figure $3 \mathrm{C}$ and Table 1). These results indicated that the $\mathrm{rBm} \alpha \mathrm{TX} 47$ from the expression vector $\mathrm{pET}-14 \mathrm{~b}$ was not a potent modulator for sodium channels examined above. However, whether toxin BmaTX47 was a potent modulator remained unclear.

In our previous work on the recombinant BmaTX14, the different pharmacological activities by different expression vectors were observed. The recombinant BmaTX14 expressed in Pichia pastoris could block sodium channel currents without affecting the gating kinetics in mouse trigeminal 
root ganglion neurons [35]. However, the recombinant BmaTX14 produced by the expression vector $\mathrm{pET}-28 \mathrm{a}$ could inhibit the fast inactivation of $\mathrm{mNa}_{\mathrm{v}} 1.4$ sodium channels with the $\mathrm{EC}_{50}$ of $82.3 \pm 15.7 \mathrm{Nm}$ [20]. Importantly, both pET-14b and pET-28a vectors are sometimes used for the expression of different $\alpha$-scorpion toxins [15,20,24]. These developments suggested the potential effects of the expression vectors and production strategies on toxin activities, and prompted us to further investigate the pharmacological properties of $\mathrm{rBm} \alpha \mathrm{TX} 47$ using the expression vector $\mathrm{pET}-28 \mathrm{a}$.

Figure 3. Pharmacological properties of $\mathrm{rBm} \alpha \mathrm{TX} 47$ on $\mathrm{rNa}_{\mathrm{v}} 1.2, \mathrm{mNa}_{\mathrm{v}} 1.4$ and $\mathrm{hNa}_{\mathrm{v}} 1.5$ channels. (A) $\mathrm{rNa}_{\mathrm{v}} 1.2$ current traces in the absence (control) or presence of $10 \mu \mathrm{M}$ rBmaTX47 from the expression vector pET-14b; (B) $\mathrm{mNa}_{\mathrm{v}} 1.4$ current traces in the absence (control) or presence of $10 \mu \mathrm{M}$ rBmaTX47 from the expression vector $\mathrm{pET}-14 \mathrm{~b}$; (C) $\mathrm{hNa}_{\mathrm{v}} 1.5$ current traces in the absence (control) or presence of $10 \mu \mathrm{M} \mathrm{rBm \alpha TX} 47$ from the expression vector $\mathrm{pET}-14 \mathrm{~b}$.
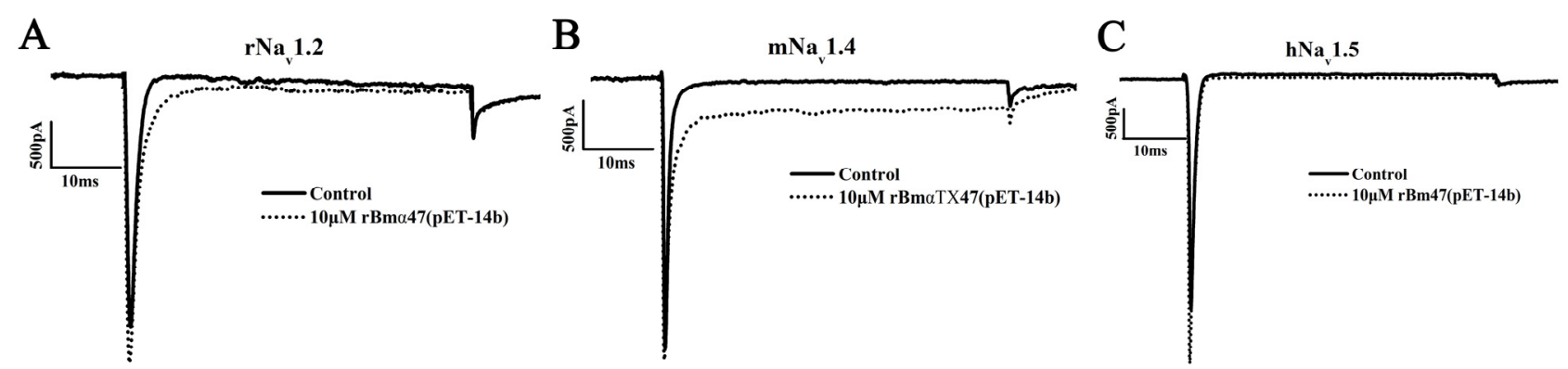

Table 1. Effects of $\mathrm{rBm} \alpha \mathrm{TX} 47$ on $\mathrm{rNa}_{\mathrm{v}} 1.2, \mathrm{mNa}_{\mathrm{v}} 1.4$ and $\mathrm{hNa}_{\mathrm{v}} 1.5$ channels. Each value represents mean $\pm \mathrm{SE}(n \geq 3)$.

\begin{tabular}{|c|c|c|c|}
\hline Toxins $(10 \mu \mathrm{M})$ & $I_{5 \mathrm{~ms}} / I_{\text {peak }}$ on $\mathrm{rNa}_{\mathrm{v}} 1.2(\%)$ & $I_{5 \mathrm{~ms}} / I_{\text {peak }}$ on $\mathrm{mNa}_{\mathrm{v}} 1.4(\%)$ & $I_{5 \mathrm{~ms}} / I_{\text {peak }}$ on hNa $1.5(\%)$ \\
\hline rBmaTX47 (pET-14b) & $11.33 \pm 1.46$ & $15.96 \pm 1.87$ & $5.04 \pm 2.38$ \\
\hline rBmaTX47 (pET-28a) & $44.12 \pm 3.17$ & $25.40 \pm 4.89$ & $65.34 \pm 3.86$ \\
\hline
\end{tabular}

\subsection{Preparation and Pharmacological Features of rBm $\alpha$ TX47 Using Expression Vector pET-28a}

According to the toxin production procedure above, $\mathrm{rBm} \alpha \mathrm{TX} 47$ was also successfully obtained using the expression vector $\mathrm{pET}-28 \mathrm{a}$, with the identical sequence to the toxin from the expression $\mathrm{pET}-14 \mathrm{~b}$ (Figure 4A). As shown in Figure 4B, the peak at 20 min corresponding to the refolded rBmaTX47 was collected manually during the RP-HPLC separation and then analyzed by the SDS-PAGE, which showed only one discernible band at about $9.5 \mathrm{kDa}$ (Figure 4B). The MALDI-TOF-MS showed the molecular weight of 9436.2 Da, which was a little different from the calculated 9416.3 Da, probably due to some post-translational processing (Figure 4C). Different from the activity of rBm $\alpha \mathrm{TX} 47$ from the expression vector $\mathrm{pET}-14 \mathrm{~b}, \mathrm{rBm} \alpha \mathrm{TX} 47$ from the expression vector $\mathrm{pET}-28 \mathrm{a}$ was more potent for $\mathrm{rNa}_{\mathrm{v}} 1.2, \mathrm{mNa}_{\mathrm{v}} 1.4$ and $\mathrm{hNa}_{\mathrm{v}} 1.5$ channels. When $10 \mu \mathrm{M} \mathrm{rBm} \alpha \mathrm{TX} 47$ from $\mathrm{pET}-28 \mathrm{a}$ was applied, the fast inactivation of sodium channel currents was inhibited, with the $I_{5 \mathrm{~ms}} / I_{\text {peak }}$ values of $44.12 \% \pm 3.17 \%$, $25.40 \% \pm 4.89 \%$ and $65.34 \% \pm 3.86 \%$ for $\mathrm{rNa}_{\mathrm{v}} 1.2, \mathrm{mNa}_{\mathrm{v}} 1.4$ and $\mathrm{hNa}_{\mathrm{v}} 1.5$ channels, respectively (Figure 4D-F, Table 1). These data showed that the expression vector pET-28a could improve the activity of the scorpion toxin rBmaTX47. 
Figure 4. Preparation and pharmacological features of rBmaTX47 from pET-28a vector. (A) Construction of expression vector BmaTX47-pET-28a. The nucleotide sequence encoding mature BmaTX47 peptide was inserted between NdeI and BamHI sites of the vector pET-28a downstream of the His 6 -tag and thrombin cleavage sequence. The full sequence of toxin rBmaTX47 was MGSSHHHHHHSSGLVPRGSHM-BmaTX47; (B) Purification of rBmaTX47 from pET-28a vector by RP-HPLC together with the tricine-SDS-PAGE analysis; $(\mathbf{C})$ Mass spectrum of rBm $\alpha$ TX47 from the MALDI-TOF-MS. The calculated molecular weight was $9416.3 \mathrm{Da}$, and the measured value was 9436.2 Da; (D) $\mathrm{rNa}_{\mathrm{v}} 1.2$ current traces in the absence (control) or presence of $10 \mu \mathrm{M} \mathrm{rBm} \alpha \mathrm{TX} 47$ produced from the expression vector $\mathrm{pET}-28 \mathrm{a}$; (E) $\mathrm{mNa}_{\mathrm{v}} 1.4$ current traces in the absence (control) or presence of $10 \mu \mathrm{M} \mathrm{rBm} \alpha \mathrm{TX} 47$ produced from the expression vector $\mathrm{pET}-28 \mathrm{a}$; (F) $\mathrm{hNa}_{\mathrm{v}} 1.5$ current traces in the absence (control) or presence of $10 \mu \mathrm{M} \mathrm{rBm \alpha TX47}$ produced from the expression vector $\mathrm{pET}-28 \mathrm{a}$.
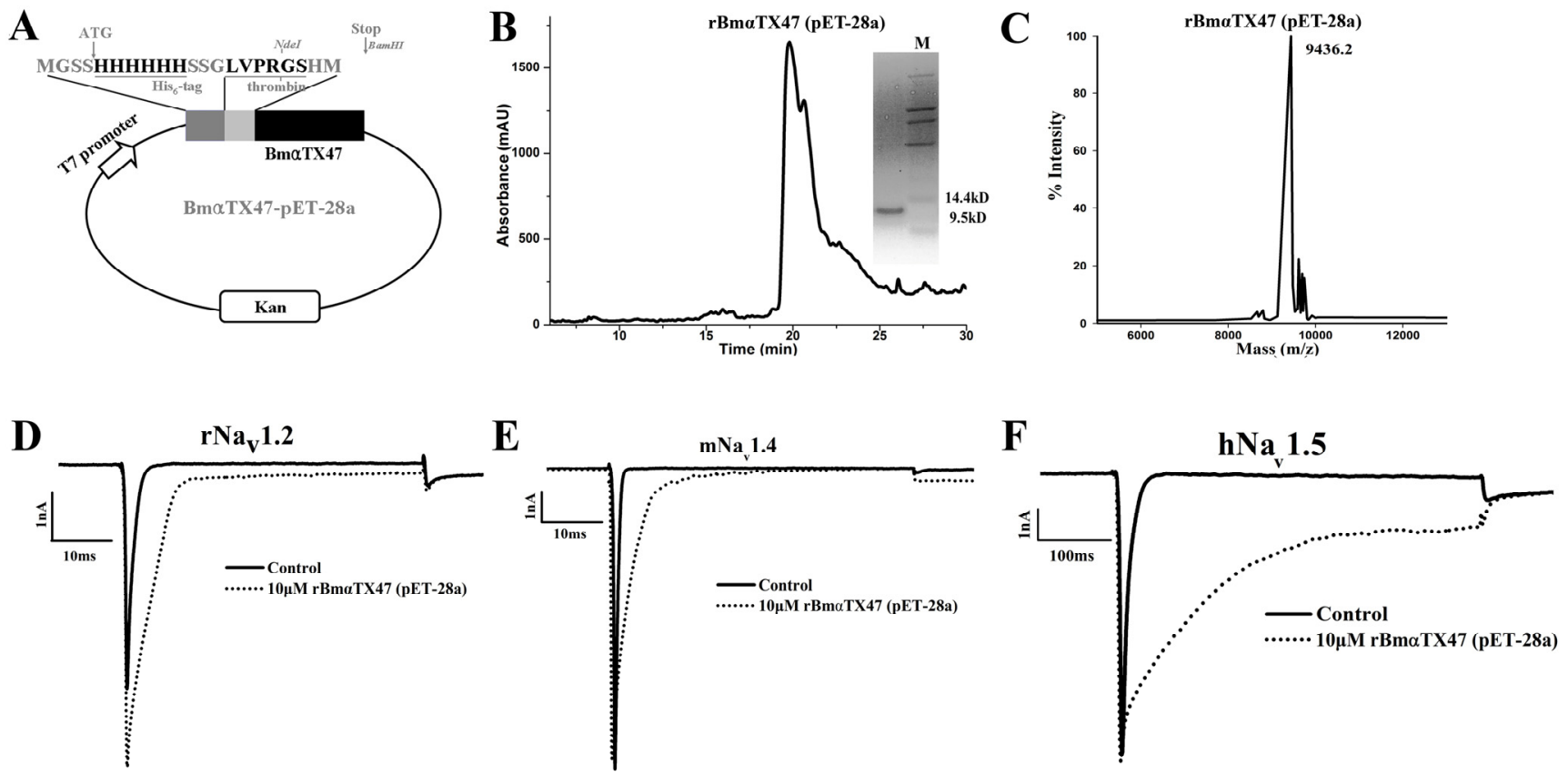

\subsection{The Pivotal Role of Expression Vectors in Scorpion Toxin Pharmacological Properties}

In order to further investigate the effect of the expression vector on the pharmacological activities of rBmaTX47 toxin, the dose-response of rBmaTX47 was conducted thereafter. For rBm $\alpha \mathrm{TX} 47$ toxin from the expression vector $\mathrm{pET}-14 \mathrm{~b}, 100 \mu \mathrm{M}$ toxins displayed the little selectivity and weak potency among $\mathrm{rNa}_{\mathrm{v}} 1.2, \mathrm{mNa}_{\mathrm{v}} 1.4$ and $\mathrm{hNa}_{\mathrm{v}} 1.5$ channels, with the $I_{5 \mathrm{~ms}} / I_{\text {peak }}$ values of about $30 \%$ for all these channels (Figure 5A). However, the $100 \mu \mathrm{M}$ rBmaTX47 toxin from the expression vector pET-28a could more significantly inhibit the fast inactivation of $\mathrm{rNa}_{\mathrm{v}} 1.2$ with $I_{5 \mathrm{~ms}} / I_{\text {peak }}$ value of about $70 \%$. The corresponding $\mathrm{EC}_{50}$ value was $7262.9 \pm 755.9 \mathrm{nM}$ through fitting with the Hill equation (Figure 5B). As for the $\mathrm{mNa}_{\mathrm{v}} 1.4$ channel, expression vector pET-28a also improved the activity of rBmaTX47, and $I_{5 \mathrm{~ms}} / I_{\text {peak }}$ value raised to about $50 \%$ for $\mathrm{mNa}_{\mathrm{v}} 1.4$ channel in the presence of $100 \mu \mathrm{M} \mathrm{rBm \alpha TX} 47$ (Figure 5B). Besides, the $100 \mu \mathrm{M} \mathrm{rBm} \alpha \mathrm{TX} 47$ from the expression vector pET-28a could efficiently delay the fast inactivation of $\mathrm{hNa}_{\mathrm{v}} 1.5$ with the $I_{5 \mathrm{~ms}} / I_{\text {peak }}$ value of about $75 \%$, and the corresponding $\mathrm{EC}_{50}$ 
value was $1005.8 \pm 118.6 \mathrm{nM}$. Importantly, it could be noted that $\mathrm{rBm} \alpha \mathrm{TX} 47$ showed preference for $\mathrm{rNa}_{\mathrm{v}} 1.2$ and $\mathrm{hNa}_{\mathrm{v}} 1.5$ channels to $\mathrm{mNa}_{\mathrm{v}} 1.4$ channel, which usually happened for the classical $\alpha$-toxins predominantly acting on the mammalian VGSCs [9,10,15,16,29-31,34,36]. Overall, the different activities and selectivities of rBmaTX47 from the expression vectors pET-14b and pET-28a indicated the critical role of the expression vectors in the scorpion toxin pharmacological properties. In addition, the pharmacological activities of rBmaTX47 further supported the function of the nearly identical Makatoxin-1 (Figure 1B), which exhibited nitrergic action in rat anococcygeus muscle expressing $\mathrm{Na}_{\mathrm{v}} 1.4$ and $\mathrm{Na}_{\mathrm{v}} 1.5$ channels $[28,37]$.

Figure 5. Pharmacological properties of rBmaTX47 from the different expression vectors on $\mathrm{rNa}_{\mathrm{v}} 1.2, \mathrm{mNa}_{\mathrm{v}} 1.4$ and $\mathrm{hNa}_{\mathrm{v}} 1.5$ channels. (A) Dose-response curves of $\mathrm{rBm} \mathrm{TX} 47$ (pET-14b) on $\mathrm{rNa}_{\mathrm{v}} 1.2, \mathrm{mNa}_{\mathrm{v}} 1.4$ and $\mathrm{hNa}_{\mathrm{v}} 1.5$ channels; (B) Dose-response curves of rBmaTX47 (pET-28a) on $\mathrm{rNa}_{\mathrm{v}} 1.2, \mathrm{mNa}_{\mathrm{v}} 1.4$ and $\mathrm{hNa}_{\mathrm{v}} 1.5$ channels. Data represent the mean $\pm \mathrm{SE}$ of at least three independent experiments.
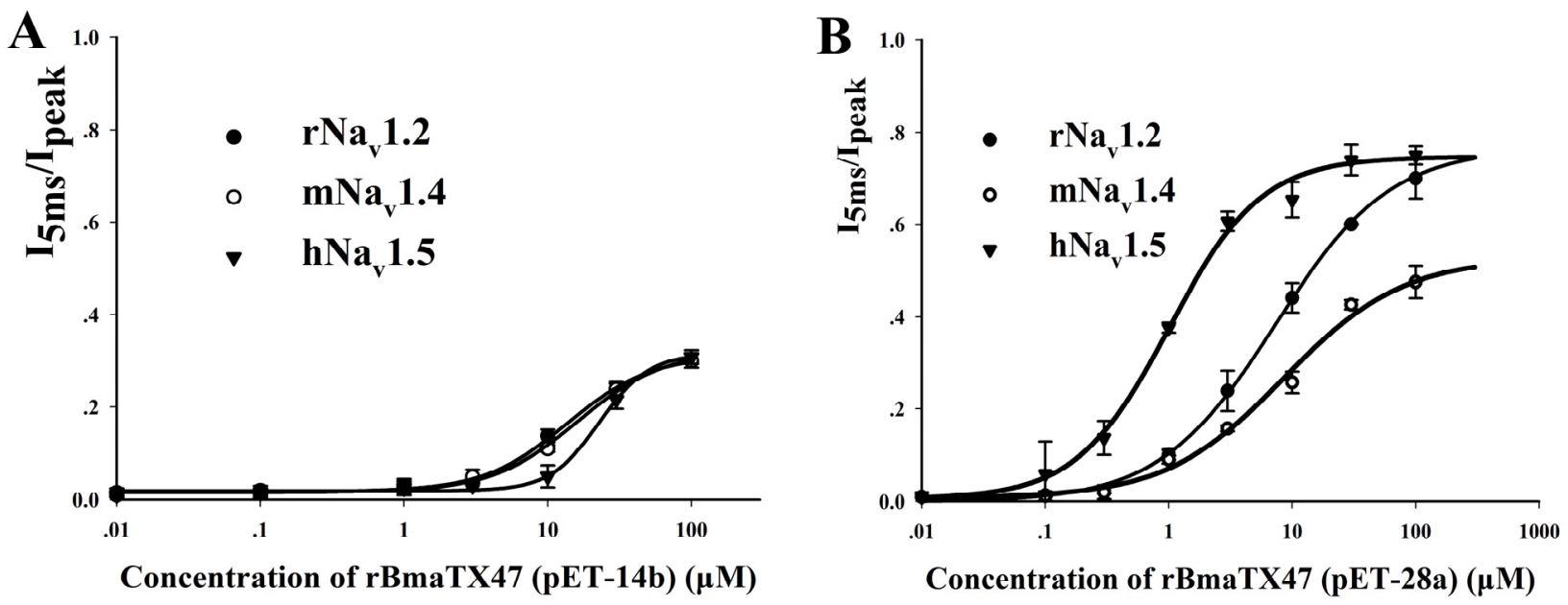

So far, the different expression vectors were used to produce $\alpha$-scorpion toxins, and their effects on the toxin pharmacology were not systematically investigated. Although the pET-14b and pET-28a vectors were often used for $\alpha$-scorpion toxins' expression, the unknown factor affecting toxin expression was also observed. For example, the pET-14b was used to express the classical $\alpha$-scorpion toxin LqhII and its mutants $[15,16,32]$. However, the $\alpha$-like toxin LqhIII was difficult to obtain by the pET-14b vector until extended by an 18-resiude apamin peptide as a linker [24]. Such phenomenon was also confirmed by our group (unpublished data). The vector pET-28a was employed for the production of the $\alpha$-scorpion toxins BmaTX14 [20], BmKIT3 [38], MeuNaTx $\alpha-12$ and MeuNaTx $\alpha-13$ [39]. Besides the expression vector $\mathrm{pET}-14 \mathrm{~b}$ and $\mathrm{pET}-28 \mathrm{a}$, other vectors were also used to express $\alpha$-scorpion toxins, such as the pET-11cK vector for expressing LqhaIT [13,32,40,41] and LqhIT2 [42], and the pVT 102 U/ $\alpha$ vector for BmKM1 and its mutants $[18,19,43]$. Significantly different from the successful expression of many scorpion toxins (with 30 40 residues) specific for the potassium channels by the pGEX-6p-1 vector [21-23], these various vectors suggested the huge difficulty in expressing $\alpha$-scorpion toxins due to some unknown factors. Therefore, the greater effort is critical for expressing the same $\alpha$-scorpion toxins by different expression vectors in the near future. These efforts would undoubtedly accelerate toxin research and potential application as the molecular tools and prospective drugs. 


\section{Experimental Section}

\section{1. cDNA Library Construction and Screening}

The venom gland cDNA library of the scorpion $B$. martensii was constructed as described previously $[25,44,45]$. Random colonies were selected for sequencing using the ABI 3730 automated sequencer. Open reading frames (ORFs) of the sequences were characterized using the ORF Finder (NCBI, Bethesda, MD, USA, http://www.ncbi.nlm.nih.gov/projects/gorf/). Signal peptides were removed using the SignalP 4.0 Server (Center for Biological Sequence Analysis, Lyngby, Denmark, http://www.cbs.dtu.dk/services/SignalP/). All sequence alignments were performed using GeneDoc software (National Resource for Biomedical Supercomputing, Pittsburgh, PA, USA) followed by manual adjustment. Sequences of $\alpha$-scorpion toxins were obtained by searching our cDNA libraries and the GenBank National Center for Biotechnology Information database (NCBI, Bethesda, MD, USA) using the Basic Local Alignment Search Tool algorithm.

\subsection{Materials}

Expression plasmids containing the cDNA encoding the rat brain sodium channel $\alpha$-subunit rSCN2A $\left(\mathrm{pNaG}_{2}\right)$, the mouse skeletal muscle sodium channel $\alpha$-subunit mSCN4A (pcDNA3.0-mH $\mathrm{m}_{2}$ ) and the human heart sodium channel $\alpha$-subnit hSCN5A (pCDNA3.1(+)- $\mathrm{hH}_{1}$ )were kindly provided by Dr. Alan L. Goldin (University of California, Irvine, CA, USA), Dr. Thomas Zimmer (Friedrich Schiller University of Jena, Jena, Germany) and Dr. Songping Liang (Hunan Normal University, Hunan, China), respectively.

\subsection{Construction of Expression Vectors}

We used the cDNA sequence of BmaTX47 from the scorpion B. martensii venom glands cDNA library as the template for polymerase chain reaction (PCR). The PCR product was digested with NdeI and BamHI and inserted into the expression vector pET-14b and pET-28a, respectively. After verification through DNA sequencing, the plasmids Bm $\alpha \mathrm{TX} 47$ from both vectors were transformed into Escherichia coli Rosetta (DE3) cells for expression.

\subsection{Expression and Purification of BmaTX47}

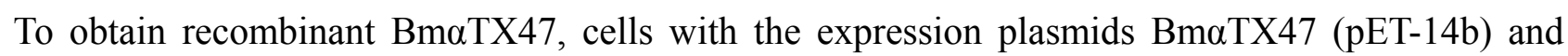
BmaTX47 (pET-28a) were incubated and induced with $0.5 \mathrm{mM}$ IPTG in LB medium as previously described [20]. Recombinant BmaTX47 protein was expressed in inclusion bodies and then denatured and refolded in 100-fold volume of $0.2 \mathrm{M}$ ammonium acetate at $16{ }^{\circ} \mathrm{C}$. The refolded protein was ultrafiltered and purified by HPLC on a C18 column $(10.0 \mathrm{~mm} \times 250 \mathrm{~mm}, 5 \mu \mathrm{m}$; Elite-HPLC, Dalian, China). The fraction containing recombinant BmaTX47 was eluted 20-21 min after injection and was further analyzed by matrix-assisted laser desorption ionization time-of-flight mass spectrometry (MALDI- TOF-MS; Voyager-DESTR, Applied Biosystems, Foster City, CA, USA). 


\subsection{Electrophysiology}

HEK293 cells were maintained in Dulbecco's modified Eagle's medium (Gibco, Grand Island, NY, USA), supplemented with $10 \%$ heat-inactivated fetal calf serum, $100 \mathrm{U} / \mathrm{mL}$ ampicillin, and $100 \mu \mathrm{g} / \mathrm{mL}$ streptomycin, in a $5 \% \mathrm{CO}_{2}$ incubator at $37^{\circ} \mathrm{C}$. They were transiently transfected with a $1: 2$ ratio of the sodium channel expression plasmids and a vector encoding enhanced green fluorescent protein (EGFP) using the TurboFect in vitro Transfection Reagent (Thermo Scientific, Pittsburgh, PA. USA). Sodium channel currents were recorded 2 days afterwards in the EGFP-positive cells.

Whole-cell patch-clamp experiments were performed at $25^{\circ} \mathrm{C}$. Data were recorded with an EPC-10 patch-clamp amplifier (HEKA Elektronik, Lambrecht, Germany) interfaced to a computer running acquisition and analysis software Pulse (HEKA Elektronik, Lambrecht, Germany). The patch pipettes contained $35 \mathrm{mM} \mathrm{NaCl}, 105 \mathrm{mM} \mathrm{CsF}, 10 \mathrm{mM}$ EGTA, and $10 \mathrm{mM}$ HEPES (pH adjusted to 7.4 with $\mathrm{CsOH}$ ). The bath solution contained $150 \mathrm{mM} \mathrm{NaCl}, 2 \mathrm{mM} \mathrm{KCl}, 1.5 \mathrm{mM} \mathrm{CaCl}_{2}, 1 \mathrm{mM} \mathrm{MgCl} 2,2 \mathrm{mM}$ $\mathrm{Na}_{2} \mathrm{ATP}$, and $10 \mathrm{mM}$ HEPES (pH adjusted to 7.4 with $\mathrm{NaOH}$ ). Peptides were dissolved in the bath solution containing $1 \%$ bovine serum albumin and then applied at above-mentioned concentrations.

To measure toxin-induced inhibition of fast inactivation, currents were elicited by depolarizing to $0 \mathrm{mV}$ from the holding potential of $-100 \mathrm{mV}$. Both the peak currents and the mean currents between 4.5 and $5 \mathrm{~ms}$ after depolarization were recorded in the absence and the presence of Bm $\alpha \mathrm{TX} 47$ peptides. The ratio $I_{5 \mathrm{~ms}} / I_{\text {peak }}$ indicates the proportion of active sodium channels whose necessary conformational changes for fast inactivation were impaired by the toxin. The concentration dependence of this toxin-induced inhibition was calculated by plotting $I_{5 \mathrm{~ms}} / I_{\text {peak }}$ as a function of toxin concentrations and fitted with the Hill equation as follows:

$$
\frac{I_{5 \mathrm{~ms}}}{I_{\text {peak }}}=a_{0}+\frac{a_{1}-a_{0}}{1+\left(\frac{E C_{50}}{[\text { Toxin }]_{\mathrm{c}}}\right)^{\mathrm{H}}}
$$

Here, $\mathrm{H}$ is the Hill coefficient; $a_{1}-a_{0}$ represents the recorded maximal effect of toxin-induced inhibition at saturating concentrations; $a_{0}$ is the measured value before applying the toxin; [Toxin $]_{\mathrm{c}}$ is the toxin concentration; and $E C_{50}$ represents the toxin concentration at the half-maximal effect. Data analysis was performed using SigmaPlot. All data are shown as arithmetic mean $\pm \mathrm{SE}$.

\section{Conclusions}

In this work, we cloned and expressed a novel $\alpha$-scorpion toxin $\operatorname{rBm} \alpha \mathrm{TX} 47$ from the scorpion Buthus martensii Karsch. The two expression vectors pET-14b and pET-28a were found to remarkably affect the toxin pharmacological activities. The toxin rBmaTX47 from the vector pET-14b slightly inhibited the fast inactivation of $\mathrm{rNa}_{\mathrm{v}} 1.2, \mathrm{mNa}_{\mathrm{v}} 1.4$ and $\mathrm{hNa}_{\mathrm{v}} 1.5$ channels; however, $\mathrm{rBm} \alpha \mathrm{TX} 47$ from the pET-28a vector significantly inhibited the fast inactivation of these three sodium channels. For $\mathrm{rNa}_{\mathrm{v}} 1.2$ and hNav1.5 channels, the $E C_{50}$ values of rBmaTX47 from the pET-28a vector were $7262.9 \pm 755.9 \mathrm{nM}$ and $1005.8 \pm 118.6 \mathrm{nM}$, respectively, which were more potent than those of toxin from the pET-14b. Besides the different activities, the selectivity of $\mathrm{rBm \alpha TX} 47$ was also influenced by the expression vectors. The toxin rBmaTX47 from pET-28a vector showed preference for $\mathrm{rNa}_{\mathrm{v}} 1.2$ and $\mathrm{hNa}_{\mathrm{v}} 1.5$ channels to mNav1.4 channel, while the toxin from $\mathrm{pET}-14 \mathrm{~b}$ vector showed little selectivity among the 
three sodium channel isoforms. These findings not only highlighted the important role of expression vectors on scorpion toxin function, but would also accelerate studies on gene engineering and future applications of scorpion toxins acting on sodium channels.

\section{Acknowledgments}

This work was supported by grants from the National Basic Research Program of China (2010CB529800), National High Technology Research and Development Program of China (2012AA020304), National Natural Sciences Foundation of China (No. 31170789), and New Century Excellent Talents in Wuhan University by the Ministry of Education of China (No. NCET-10-0651).

\section{Conflicts of Interest}

The authors declare no conflict of interest.

\section{References}

1. Catterall, W.A. Voltage-gated sodium channels at 60: Structure, function and pathophysiology. J. Physiol. 2012, 590, 2577-2589.

2. Catterall, W.A.; Goldin, A.L.; Waxman, S.G. International Union of Pharmacology. XLVII. Nomenclature and structure-function relationships of voltage-gated sodium channels. Pharmacol. Rev. 2005, 57, 397-409.

3. Beneski, D.A.; Catterall, W.A. Covalent labeling of protein components of the sodium channel with a photoactivable derivative of scorpion toxin. Proc. Natl. Acad. Sci. USA 1980, 77, 639-643.

4. Yang, N.; Horn, R. Evidence for voltage-dependent S4 movement in sodium channels. Neuron 1995, 15, 213-218.

5. Yang, N.; George, A.L., Jr.; Horn, R. Molecular basis of charge movement in voltage-gated sodium channels. Neuron 1996, 16, 113-122.

6. Chanda, B.; Bezanilla, F. Tracking voltage-dependent conformational changes in skeletal muscle sodium channel during activation. J. Gen. Physiol. 2002, 120, 629-645.

7. Catterall, W.A.; Cestele, S.; Yarov-Yarovoy, V.; Yu, F.H.; Konoki, K.; Scheuer, T. Voltage-gated ion channels and gating modifier toxins. Toxicon 2007, 49, 124-141.

8. Bosmans, F.; Tytgat, J. Voltage-gated sodium channel modulation by scorpion alpha-toxins. Toxicon 2007, 49, 142-158.

9. Chen, H.; Gordon, D.; Heinemann, S.H. Modulation of cloned skeletal muscle sodium channels by the scorpion toxins Lqh II, Lqh III, and Lqh alphaIT. Pflugers Arch. 2000, 439, 423-432.

10. Leipold, E.; Lu, S.; Gordon, D.; Hansel, A.; Heinemann, S.H. Combinatorial interaction of scorpion toxins Lqh-2, Lqh-3, and LqhalphaIT with sodium channel receptor sites-3. Mol. Pharmacol. 2004, 65, 685-691.

11. Gilles, N.; Blanchet, C.; Shichor, I.; Zaninetti, M.; Lotan, I.; Bertrand, D.; Gordon, D. A scorpion alpha-like toxin that is active on insects and mammals reveals an unexpected specificity and distribution of sodium channel subtypes in rat brain neurons. J. Neurosci. 1999, 19, 8730-8739. 
12. Krimm, I.; Gilles, N.; Sautiere, P.; Stankiewicz, M.; Pelhate, M.; Gordon, D.; Lancelin, J.M. NMR structures and activity of a novel alpha-like toxin from the scorpion Leiurus quinquestriatus hebraeus. J. Mol. Biol. 1999, 285, 1749-1763.

13. Zilberberg, N.; Froy, O.; Loret, E.; Cestele, S.; Arad, D.; Gordon, D.; Gurevitz, M. Identification of structural elements of a scorpion alpha-neurotoxin important for receptor site recognition. J. Biol. Chem. 1997, 272, 14810-14816.

14. Chen, H.; Heinemann, S.H. Interaction of scorpion alpha-toxins with cardiac sodium channels: Binding properties and enhancement of slow inactivation. J. Gen. Physiol. 2001, 117, 505-518.

15. Kahn, R.; Karbat, I.; Ilan, N.; Cohen, L.; Sokolov, S.; Catterall, W.A.; Gordon, D.; Gurevitz, M. Molecular requirements for recognition of brain voltage-gated sodium channels by scorpion alpha-toxins. J. Biol. Chem. 2009, 284, 20684-20691.

16. Gur, M.; Kahn, R.; Karbat, I.; Regev, N.; Wang, J.; Catterall, W.A.; Gordon, D.; Gurevitz, M. Elucidation of the molecular basis of selective recognition uncovers the interaction site for the core domain of scorpion alpha-toxins on sodium channels. J. Biol. Chem. 2011, 286, 35209-35217.

17. Wang, J.; Yarov-Yarovoy, V.; Kahn, R.; Gordon, D.; Gurevitz, M.; Scheuer, T.; Catterall, W.A. Mapping the receptor site for alpha-scorpion toxins on a $\mathrm{Na}^{+}$channel voltage sensor. Proc. Natl. Acad. Sci. USA 2011, 108, 15426-15431.

18. Sun, Y.M.; Bosmans, F.; Zhu, R.H.; Goudet, C.; Xiong, Y.M.; Tytgat, J.; Wang, D.C. Importance of the conserved aromatic residues in the scorpion alpha-like toxin BmK M1: The hydrophobic surface region revisited. J. Biol. Chem. 2003, 278, 24125-24131.

19. Wang, C.G.; Gilles, N.; Hamon, A.; Le Gall, F.; Stankiewicz, M.; Pelhate, M.; Xiong, Y.M.; Wang, D.C.; Chi, C.W. Exploration of the functional site of a scorpion alpha-like toxin by site-directed mutagenesis. Biochemistry 2003, 42, 4699-4708.

20. Dai, H.; Yin, S.; Li, T.; Cao, Z.; Ji, Y.; Wu, Y.; Li, W. Recombinant expression, purification, and characterization of scorpion toxin BmalphaTX14. Protein Expr. Purif. 2012, 82, 325-331.

21. Han, S.; Yi, H.; Yin, S.J.; Chen, Z.Y.; Liu, H.; Cao, Z.J.; Wu, Y.L.; Li, W.X. Structural basis of a potent peptide inhibitor designed for Kv1.3 channel, a therapeutic target of autoimmune disease. J. Biol. Chem. 2008, 283, 19058-19065.

22. Chen, Z.; Hu, Y.; Han, S.; Yin, S.; He, Y.; Wu, Y.; Cao, Z.; Li, W. ImKTx1, a new Kv1.3 channel blocker with a unique primary structure. J. Biochem. Mol. Toxicol. 2011, 25, 244-251.

23. Chen, Z.Y.; Zeng, D.Y.; Hu, Y.T.; He, Y.W.; Pan, N.; Ding, J.P.; Cao, Z.J.; Liu, M.L.; Li, W.X.; $\mathrm{Yi}, \mathrm{H}$; et al. Structural and functional diversity of acidic scorpion potassium channel toxins. PLoS One 2012, 7, e35154.

24. Karbat, I.; Kahn, R.; Cohen, L.; Ilan, N.; Gilles, N.; Corzo, G.; Froy, O.; Gur, M.; Albrecht, G.; Heinemann, S.H.; et al. The unique pharmacology of the scorpion alpha-like toxin Lqh3 is associated with its flexible C-tail. FEBS J. 2007, 274, 1918-1931.

25. Li, S.; Ma, Y.; Jang, S.; Wu, Y.; Liu, H.; Cao, Z.; Li, W. A HindIII BAC library construction of Mesobuthus martensii Karsch (Scorpiones:Buthidae): An important genetic resource for comparative genomics and phylogenetic analysis. Genes Genet. Syst. 2009, 84, 417-424.

26. Ma, Y.; Zhao, R.; He, Y.; Li, S.; Liu, J.; Wu, Y.; Cao, Z.; Li, W. Transcriptome analysis of the venom gland of the scorpion Scorpiops jendeki: Implication for the evolution of the scorpion venom arsenal. BMC Genomics 2009, 10, 290. 
27. He, Y.; Zhao, R.; Di, Z.; Li, Z.; Xu, X.; Hong, W.; Wu, Y.; Zhao, H.; Li, W.; Cao, Z. Molecular diversity of Chaerilidae venom peptides reveals the dynamic evolution of scorpion venom components from Buthidae to non-Buthidae. J. Proteomics 2013, 89, 1-14.

28. Gong, J.; Kini, R.M.; Gwee, M.C.; Gopalakrishnakone, P.; Chung, M.C. Makatoxin I, a novel toxin isolated from the venom of the scorpion Buthus martensi Karsch, exhibits nitrergic actions. J. Biol. Chem. 1997, 272, 8320-8324.

29. Chen, H.; Lu, S.; Leipold, E.; Gordon, D.; Hansel, A.; Heinemann, S.H. Differential sensitivity of sodium channels from the central and peripheral nervous system to the scorpion toxins Lqh-2 and Lqh-3. Eur. J. Neurosci. 2002, 16, 767-770.

30. Liu, L.H.; Bosmans, F.; Maertens, C.; Zhu, R.H.; Wang, D.C.; Tytgat, J. Molecular basis of the mammalian potency of the scorpion alpha-like toxin, BmK M1. FASEB J. 2005, 19, 594-596.

31. Ye, X.; Bosmans, F.; Li, C.; Zhang, Y.; Wang, D.C.; Tytgat, J. Structural basis for the voltage-gated $\mathrm{Na}^{+}$channel selectivity of the scorpion alpha-like toxin BmK M1. J. Mol. Biol. 2005, 353, 788-803.

32. Weinberger, H.; Moran, Y.; Gordon, D.; Turkov, M.; Kahn, R.; Gurevitz, M. Positions under positive selection--key for selectivity and potency of scorpion alpha-toxins. Mol. Biol. Evol. 2010, 27, 1025-1034.

33. Chen, R.; Chung, S.H. Binding modes and functional surface of anti-mammalian scorpion alpha-toxins to sodium channels. Biochemistry 2012, 51, 7775-7782.

34. Durek, T.; Vetter, I.; Wang, C.I.; Motin, L.; Knapp, O.; Adams, D.J.; Lewis, R.J.; Alewood, P.F. Chemical engineering and structural and pharmacological characterization of the alpha-scorpion toxin OD1. ACS Chem. Biol. 2013, 8, 1215-1222.

35. Wang, K.; Yin, S.J.; Lu, M.; Yi, H.; Dai, C.; Xu, X.J.; Cao, Z.J.; Wu, Y.L.; Li, W.X. Functional analysis of the alpha-neurotoxin, BmalphaTX14, derived from the Chinese scorpion, Buthus martensii Karsch. Biotechnol. Lett. 2006, 28, 1767-1772.

36. Zhu, S.; Peigneur, S.; Gao, B.; Lu, X.; Cao, C.; Tytgat, J. Evolutionary diversification of Mesobuthus alpha-scorpion toxins affecting sodium channels. Mol. Cell. Proteomics 2012, 11, doi:10.1074/mcp.M111.012054.

37. Zimmer, T.; Bollensdorff, C.; Haufe, V.; Birch-Hirschfeld, E.; Benndorf, K. Mouse heart $\mathrm{Na}^{+}$ channels: Primary structure and function of two isoforms and alternatively spliced variants. Am. J. Physiol.-Heart Circ. Physiol. 2002, 282, H1007-H1017.

38. Yu, J.B.; Ji, P.; Zha, X.M.; Shen, W.D.; Wu, X.F. Cloning and expression of Buthus martensii Karsch scorpion toxin gene (BmK IT3) in Escherichia coli. Sheng Wu Gong Cheng Xue Bao 2002, 18, 106-108.

39. Zhu, L.; Peigneur, S.; Gao, B.; Tytgat, J.; Zhu, S. Two recombinant alpha-like scorpion toxins from Mesobuthus eupeus with differential affinity toward insect and mammalian $\mathrm{Na}^{+}$channels. Biochimie 2013, 95, 1732-1740.

40. Zilberberg, N.; Gordon, D.; Pelhate, M.; Adams, M.E.; Norris, T.M.; Zlotkin, E.; Gurevitz, M. Functional expression and genetic alteration of an alpha scorpion neurotoxin. Biochemistry 1996, 35, 10215-10222.

41. Karbat, I.; Frolow, F.; Froy, O.; Gilles, N.; Cohen, L.; Turkov, M.; Gordon, D.; Gurevitz, M. Molecular basis of the high insecticidal potency of scorpion alpha-toxins. J. Biol. Chem. 2004, 279, 31679-31686. 
42. Karbat, I.; Turkov, M.; Cohen, L.; Kahn, R.; Gordon, D.; Gurevitz, M.; Frolow, F. X-ray structure and mutagenesis of the scorpion depressant toxin LqhIT2 reveals key determinants crucial for activity and anti-insect selectivity. J. Mol. Biol. 2007, 366, 586-601.

43. Sun, Y.M.; Liu, W.; Zhu, R.H.; Goudet, C.; Tytgat, J.; Wang, D.C. Roles of disulfide bridges in scorpion toxin BmK M1 analyzed by mutagenesis. J. Pept. Res. 2002, 60, 247-256.

44. Chen, Z.Y.; Hu, Y.T.; Yang, W.S.; He, Y.W.; Feng, J.; Wang, B.; Zhao, R.M.; Ding, J.P.; Cao, Z.J.; Li, W.X.; et al. Hg1, novel peptide inhibitor specific for Kv1.3 channels from first scorpion Kunitz-type potassium channel toxin family. J. Biol. Chem. 2012, 287, 13813-13821.

45. Zhao, R.; Dai, H.; Qiu, S.; Li, T.; He, Y.; Ma, Y.; Chen, Z.; Wu, Y.; Li, W.; Cao, Z. SdPI, the first functionally characterized Kunitz-type trypsin inhibitor from scorpion venom. PLoS One 2011, 6, e27548.

(C) 2014 by the authors; licensee MDPI, Basel, Switzerland. This article is an open access article distributed under the terms and conditions of the Creative Commons Attribution license (http://creativecommons.org/licenses/by/3.0/). 